\title{
One-Stage Endovascular Embolization for Multiple Intracranial Aneurysms
}

\author{
Teng-Fei $\mathrm{LI}^{1,2^{*}}$, Shao-Feng SHUI ${ }^{1,2^{*}}$, Xin-Wei HAN ${ }^{1,2}$, Lei YAN ${ }^{1,2}$, Ji MA ${ }^{1,2}$, Dong GUO ${ }^{1,2}$ \\ ${ }^{1}$ The First Affiliated Hospital of Zhengzhou University, Department of Interventional Radiology, Zhengzhou, China \\ ${ }^{2}$ Interventional Institute of Zhengzhou University, Zhengzhou, China \\ ${ }^{*}$ Teng-Fei LI and Shao-Feng SHUI contributed equally to this paper.
}

\section{ABSTRACT}

AIM: Clinical treatment of multiple intracranial aneurysms remains challenging due its higher rate of rupture compared to a single aneurysm. We aimed to assess the efficacy of one-stage endovascular embolization for treatment of multiple intracranial aneurysms.

MATERIAL and METHODS: We treated 72 aneurysms from 33 patients with one-stage endovascular embolization from September 2010 to October 2015. Of these, 60 aneurysms were treated with coils, while 12 wide-neck aneurysms were embolized with the assistance of intracranial stents. Follow-up studies ranged from 3 to 28 months, and patient outcomes were assessed using the modified Rankin Scale (mRS) and digital subtraction angiography (DSA).

RESULTS: All aneurysms were embolized successfully and no complications occurred. DSA immediately after procedure revealed that complete occlusion was achieved in 39 cases, neck remnant was observed in 27 cases, and a residual aneurysm remnant was observed in six cases. All patients achieved excellent clinical outcomes following embolization (27 patients with a mRS score of 0 and 6 with a mRS score of 1). Six months after the procedure, DSA was performed on 28 patients, revealing one case with a neck remnant, and two cases with completely occluded aneurysms. Despite these outcomes, there was no rupture or hemorrhage of these aneurysms throughout the six months.

CONCLUSION: One-stage endovascular embolization is a safe and effective treatment for multiple intracranial aneurysms, but long-term outcomes should be further evaluated.

KEYWORDS: Multiple intracranial aneurysms, Embolization, Endovascular, Stent, Coiling

ABBREVIATIONS: DSA: Digital subtraction angiography, mRS: modified Rankin Scale, MIA: Multiple intracranial aneurysms, SAH: Subarachnoid hemorrhage, 3D: Three-dimensional, MCA: Middle cerebral artery, CT: Computed tomography, MR: Magnetic resonance, UIA: Unruptured intracranial aneurysm.

\section{INTRODUCTION}

$\mathrm{M}$ ultiple intracranial aneurysms (MIA) are defined by the presence of two or more aneurysms in one patient. They occur in $19 \%$ to $34 \%$ of patients who present with subarachnoid hemorrhage (SAH) $(7-9,14)$. Clinical treatment of MIA is challenging as it has a higher rate of rupture compared to a single aneurysm and requires multiple operations with an increase in associated costs.

We studied the effectiveness of using one-stage endovascular embolization as an alternative to multiple operations for the treatment of MIA. 


\section{MATERIAL and METHODS}

\section{Patients}

From September 2010 to October 2015, 33 patients with multiple intracranial aneurysms were hospitalized into the First Affiliated Hospital of Zhengzhou University, and treated with one-stage endovascular embolization. Written informed consent was obtained from all the patients for the publication of this article and any accompanying images. This study was approved by the Ethics Committee of The First Affiliated Hospital of Zhengzhou University.

There were 13 men and 20 women (aged from 34 to 77 years) included in the study. Among these 33 patients, 21 patients were treated due to spontaneous $\mathrm{SAH}$, including 19 cases of first bleeding and two cases of re-bleeding. Of these 21 patients with $\mathrm{SAH}$, there were 12 cases of Hunt and Hess $(\mathrm{H} \& \mathrm{H})$ grade I (asymptomatic or mild headache), six cases of Hunt and Hess grade II (moderate to severe headache), and three cases of Hunt and Hess grade III (drowsiness/confusion). For the remaining 12 patients, aneurysms were found during a health check.

\section{Aneurysm Identification}

The 33 patients were examined prior to the procedure using digital subtraction angiography (DSA) and three-dimensional (3D) reconstruction of rotational DSA. A total of 72 aneurysms were found. 28 of the patients had two aneurysms, four patients had three, and in one patient, four aneurysms existed. There were 12 wide-necked aneurysms in total. Fifty-one aneurysms were located on the anterior circulation, including 18 in the carotid-ophthalmic section, 26 in the intracavemous carotid, and two in the internal carotid artery bifurcation. Twenty-one aneurysms were located on posterior circulation, including four in the vertebral artery, three in the basilar trunk, one in the top basilar artery, and 13 in the posterior cerebral artery. The diameters of aneurysms were as follows: $\leq 3 \mathrm{~mm}$ (17 aneurysms); 3-10 mm (42 aneurysms); and >10 mm (13 aneurysms).

\section{Identification of the Aneurysms Responsible for Bleeding}

For MIA with SAH, the aneurysm responsible for bleeding was located at the site of blood clot using a computed tomography (CT) scan (Figure 1). If no blood clot was observed on the CT image, or if the aneurysms were multiple, the biggest or most irregular shaped aneurysm was identified as the bleeding source. Also the irregular shape of the daughter sac of the aneurysm had the most predictive value for determining the bleeding source. For MIA without SAH, the aneurysm responsible for bleeding was identified when cranial nerve symptoms were in accordance with the aneurysm's location. For asymptomatic MIA, aneurysms bigger than $5 \mathrm{~mm}$ in size were presumed to be the most likely to rupture and bleed.

\section{Treatment}

All patients were treated with one-stage embolization. The procedure was done under intubation anesthesia. The number, location, size, shape and relationship with the parent artery of the aneurysms were confirmed by DSA. Rotational DSA
(3D reconstruction) was used to choose the best operating position. After heparinization, a 6 Fr guiding catheter (Cordis Neurovascular, Miami, FL, USA) was inserted into the target artery. The aneurysm responsible for bleeding was embolized first, followed by other aneurysms. Sixty narrownecked aneurysms were treated only with coils (Microplex (MicroVention, Aliso Viejo, CA, USA) or Axium (eV3, Irvine, CA,USA)), while 12 wide-necked aneurysms were embolized with the assistance of stents (9 Solitaire stents (eV3, Irvine, CA, USA) and 3 Enterprise stents (Cordis Neurovascular, Miami, FL,USA)). Among those treated using assistant stents, patients with unruptured aneurysms were given aspirin $(100 \mathrm{mg} / \mathrm{d})$ and clopidogrel $(75 \mathrm{mg} / \mathrm{d})$ for five days before intervention. For those patients with ruptured aneurysms, clopidogrel (300 $\mathrm{mg}$ ) and aspirin (300 mg) were given at least two hours, but not more than three hours, prior to embolization. For patients with impaired consciousness, medication was administered rectally after anesthesia. After the procedure, clopidogrel (75 $\mathrm{mg} / \mathrm{d}$ ) was given for six weeks, and aspirin $(100 \mathrm{mg} / \mathrm{d})$ was given long-term.

\section{Postoperative and Follow-Up Evaluation}

The degree of aneurysm occlusion was evaluated with DSA by two senior professional physicians immediately after the procedure, and then again, six months later. Modified Raymond classification was used to evaluate the patients

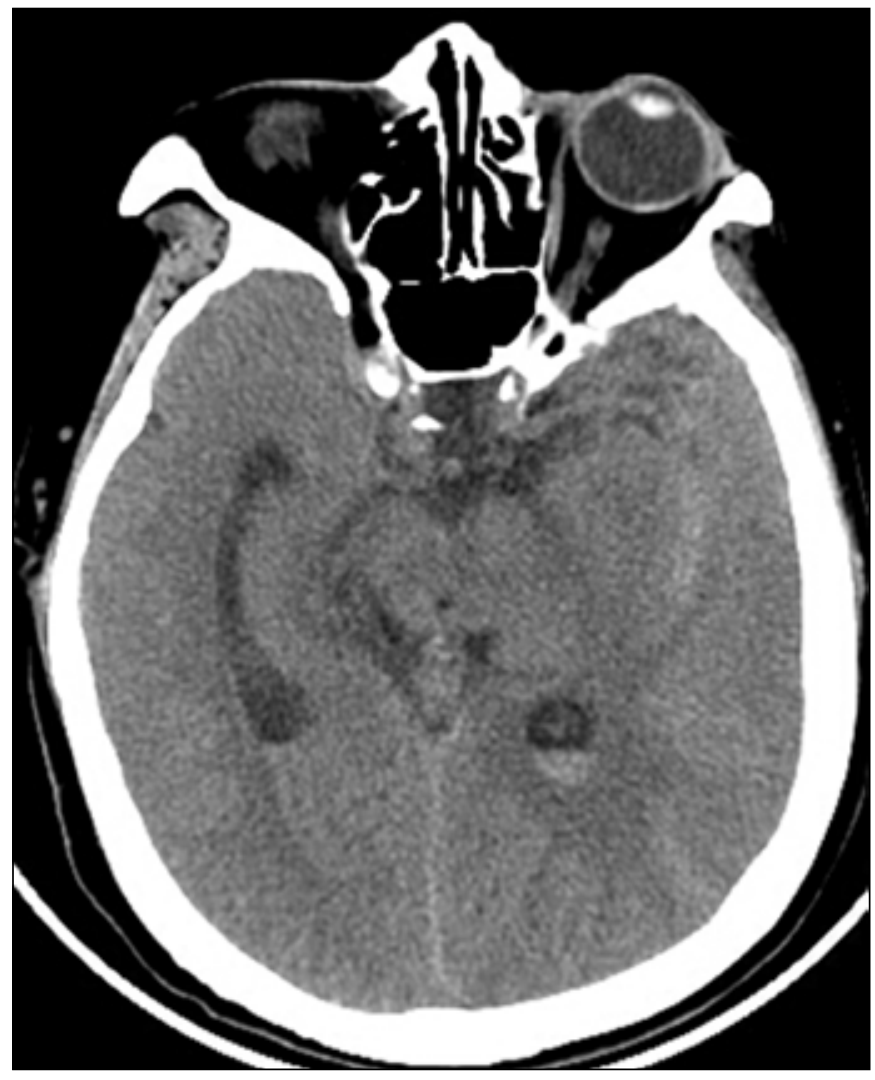

Figure 1: Head CT scan of a 62-year-old female indicating subarachnoid hemorrhage $(\mathrm{SAH})$, located mainly at the left lateral fissure. 
as follows: (i) complete occlusion, when no contrast agent appears in the intracavity of the aneurysm; (ii) neck remnant, when contrast agent appears at the neck of the aneurysm; and (iii) aneurysm remnant, when part of the aneurysm fills with contrast agent (15). The clinical outcome was assessed at discharge, and again after six months, using the modified Rankin Scale (mRS).

\section{RESULTS}

All embolization procedures were successful and all patients were discharged from hospital without permanent neural dysfunction. Immediately after the procedure, DSA showed 39 cases of aneurysms with complete occlusion, 27 with neck remnant, and 6 with aneurysm remnant. All of the stents were placed in an ideal location and no complications (such as stent occlusion, dislocation, endarterium tearing, obstruction of parent artery and its branches, aneurysm rupture or thromboembolism) occurred. An example of a one-stage embolization for three aneurysms is shown in Figure $2 \mathrm{~A}-\mathrm{H}$.

All 33 patients received follow-up for a period of 3 to 28 months. For 27 patients, a mRS score of zero was observed, while six patients scored a mRS of one. For 28 patients, we performed DSA examination six months after the procedure.
One patient showed a neck remnant (Figure 3A-D), one showed a completely occluded aneurysm located at A1 section of left middle cerebral artery (MCA), and one patient had a completely occluded aneurysm located in the basilar artery. Despite this, there was no rupture or hemorrhage of these aneurysms.

\section{DISCUSSION}

Traditionally, multiple aneurysms required more than one operation to treat them effectively. Unfortunately, the risks associated with these procedures are relatively high as remnant aneurysms are likely to rupture during multiple operations. This risk becomes increased in patients with unstable blood pressure, or if other therapies (such as volume expansion, anticoagulation, dehydration, lumbar puncture, or ventricular drainage) are used. In addition, due to the multiple operations and lengthy hospitalization time, the costs associated with treating MIA are higher. A one-stage procedure could overcome these problems, by not only preventing the rupture of aneurysms, but also avoiding malpractice resulting from misjudgment of the responsible aneurysm.

Embolizing any unruptured aneurysms in MIA is necessary as they may rupture in the future causing a cerebral bleeding.
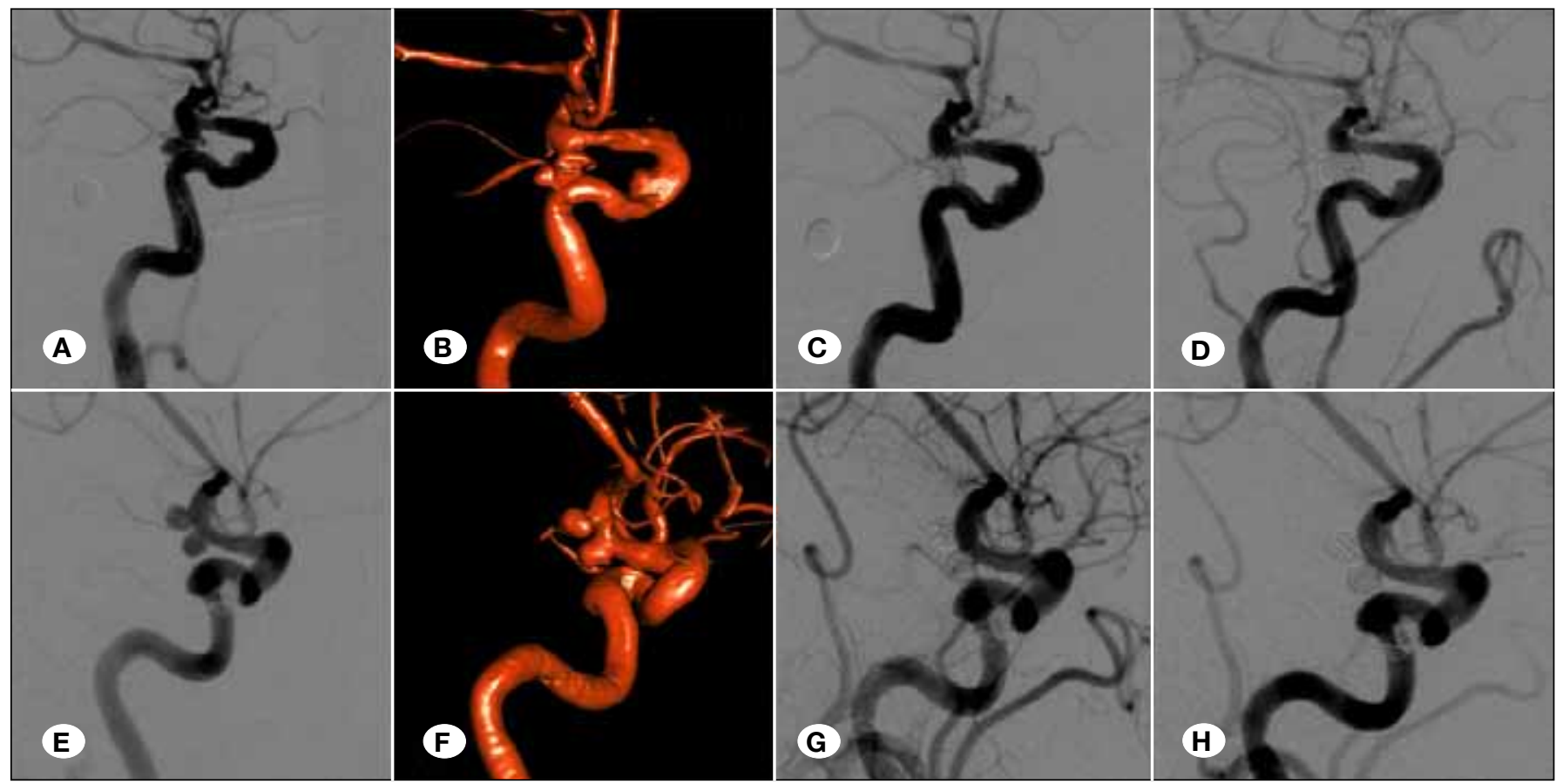

Figure 2: DSA and 3D reconstruction of a patient (62-year-old female) with multiple aneurysms described in Figure 1, who underwent one-stage embolization. Images (A) to (D) are of the left posterior communicating artery. Images (E) to (F) show the right posterior communicating artery and carotid-ophthalmic section. (A) DSA and (B) 3D reconstruction images show an irregular aneurysm (2.7 $\mathrm{mm} \times 5.4 \mathrm{~mm}$ in size) in the left posterior communicating artery. (E) DSA and (F) 3D reconstruction images show aneurysms in the right posterior communicating artery $(1.7 \mathrm{~mm} \times 3.8 \mathrm{~mm})$ and in the carotid-ophthalmic section $(1.9 \mathrm{~mm} \times 4.1 \mathrm{~mm})$. The left posterior communicating artery irregular aneurysm was considered responsible for bleeding, according to the head CT. After the insertion of a microcatheter into the location, coils were densely filled into the sac. DSA of the (C) left and (G) right posterior communicating artery and carotid-ophthalmic section immediately after the procedure showing the aneurysm was completely occluded, and the parent artery was clear. DSA of the (D) left and (H) right posterior communicating artery and carotid-ophthalmic section six months later revealing that the embolization was stable, and the parent artery was still clear. 

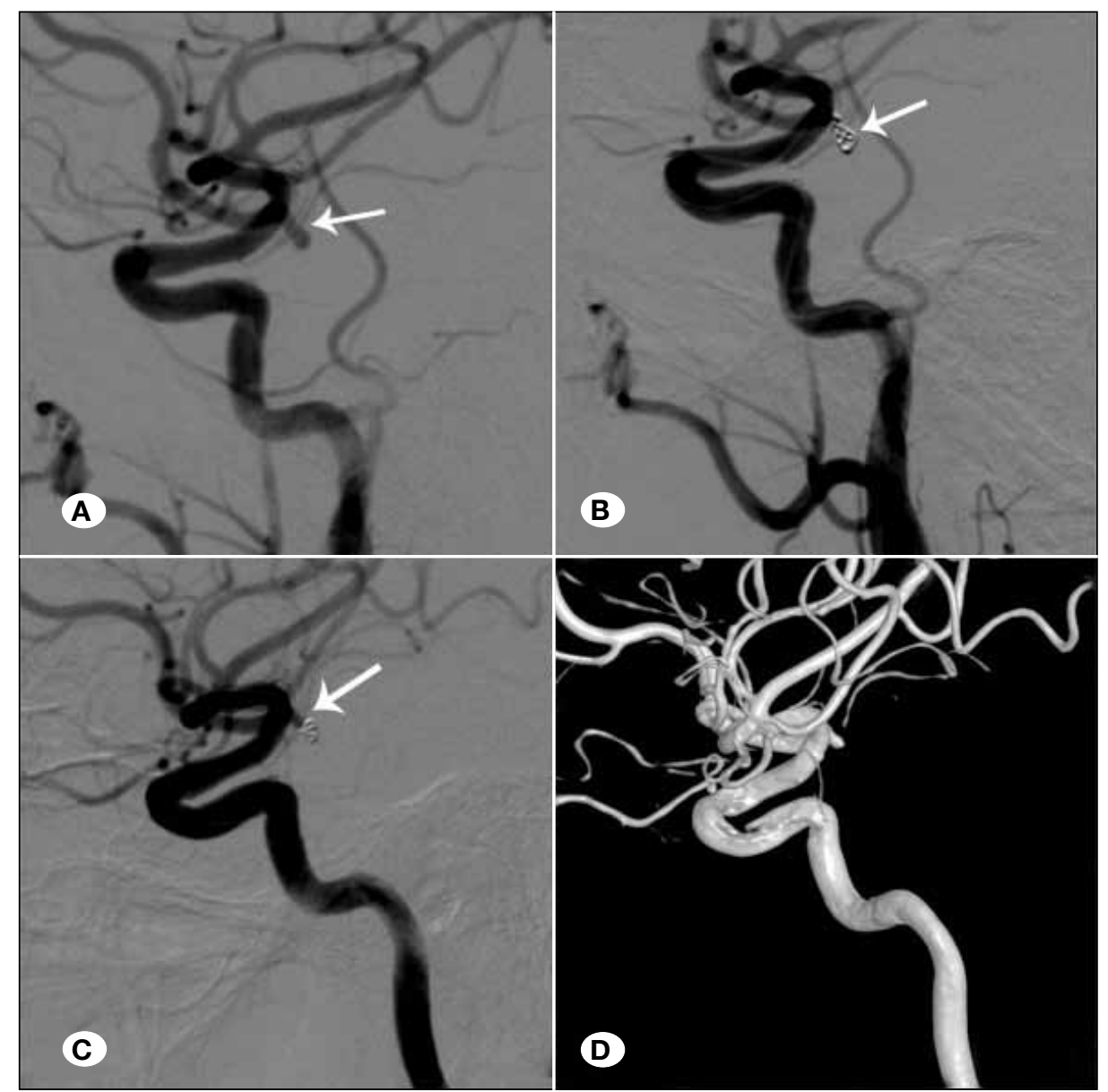

Figure 3: DSA and 3D reconstruction images of a female ( 45 years old) patient with multiple aneurysms found during a standard health check. The patient underwent a one-stage coil embolization procedure. A) Initial DSA image, showing one aneurysm in the right posterior communicating artery and one in the left A1 section. B) DSA image immediately after coil embolization, showing that the aneurysm was embolized fully and the parent artery was clear. C) DSA and D) 3D reconstruction images six months after embolization, revealing that the coils were compressed and a neck remnant

C had occurred.

Cheong et al. analyzed more than 300 cases of multiple aneurysms and revealed that patients with a history of SAH have a formerly-unruptured-aneurysms rupture rate of $0.28 \%$ to $1.63 \%$ (1). In addition, Hino et al. treated 76 cases of multiple aneurysms and in six cases, the aneurysm responsible for bleeding was not correctly determined resulting in an additional four cases of cerebral hemorrhage (2). These results support the use of a one-stage embolization procedure for multiple aneurysms to prevent future ruptures and problems arising from incorrect determination of the rupture site.

To date, there have been many reported cases where multiple aneurysms have been treated with clipping $(4,5,11-13,17)$. Clipping of multiple aneurysms in MIA, however, results in poorer outcomes than in a single aneurysm $(5,11-13,17)$. This can be explained by the increased manipulation of cerebral arteries and brain tissue during multiple aneurysm surgery $(11,13)$. Also, one-stage clipping for multiple aneurysms may be limited in cases where multiple aneurysms are distributed bilaterally, or are in both located in the anterior and posterior circulations (11).

Unlike clipping, embolizing using coils does not require much manipulation of cerebral arteries or brain tissue. Solander et al.(16) reported that one-stage embolization is safe, whether or not the multiple aneurysms are in the acute phase of bleeding. Our results are in agreement with this study; for all 72 aneurysms that underwent one-stage embolization, no procedure-related complications occurred, even if the aneurysm was bleeding. In addition, in all 33 patients, a mRS score of zero or one was recorded, indicating they were clinically asymptomatic or had no significant disabilities.

Another advantage of one-stage coiling embolization of MIA is that coiling avoids repeat general anesthesia and groin punctures compared with multiple separate procedures. Cost-effectiveness is another expected advantage. Coiling, particularly one-stage coiling, has been associated with a significantly shorter hospital stay and lower hospital expenses for patients with both ruptured and unruptured aneurysms compared to clipping procedures (3).

Despite these advantages, one-stage embolization requires a high level of skill and has its own limitations. For example, theoretically, vasospasm or vessel thromboembolism is likely to be triggered by the lengthy embolization operation with repeated intubation in a single parent artery. If rupture occurs, the procedure becomes even more difficult. In our study, the easier and distal aneurysms were embolized first, and the harder and proximal ones were treated later. This method should be employed in all one-stage embolization procedures to avoid vasospasm or intraoperative rupture of the aneurysm.

Determination of the aneurysm responsible for bleeding (i.e. the rupture site) is of significant importance in treating MIA, 
and is currently determined empirically. The appearance of the aneurysm (by CT scan and DSA) and the vasospasm of the parent artery should be comprehensive assessed prior to the embolization procedure to identify the aneurysm responsible for bleeding $(2,6)$. The influence of hemodynamics on the course of SAH should also be considered. For example, if an aneurysm's long axis is in the same direction of blood flow, and its vesicle or daughter sac is on the impact point, this aneurysm should be the one considered responsible for the bleeding. For cases where determining the aneurysm responsible for bleeding is difficult, Matouk et al. propose that high definition magnetic resonance (MR) may help (10). The wall of the ruptured aneurysm would be enhanced on the MR image due to localized vascular endothelial inflammation, and therefore, contrast agent will infiltrate into the vessel wall through the abnormal vascular endothelia. However, MR has its own problems. This method requires high level devices (i.e. operation rooms equipped with both DSA and MR machines) and a lengthy examination procedure requiring intubation anesthesia.

For patients without $\mathrm{SAH}$, whether the unruptured intracranial aneurysms (UIA) should be treated depends on H\&H scale, size and location of the aneurysm, age of patient, and relative risk factors for bleeding. Specifically;

(i). As UIA smaller than $5 \mathrm{~mm}$, a conservative treatment will be selected, at the same time we will also consider the mental burden of the patient.

(ii). Immediate treatment will be adopted if patient age $<60$ and aneurysm size $>5 \mathrm{~mm}$, the age limit will be lifted if aneurysms located at posterior circulation (especially the top of basilar artery) and posterior communicating artery, where much higher risks of bleeding were reported.

(iii). If the aneurysm size is larger than $10 \mathrm{~mm}$, the age criteria could be relaxed to 70 years.

(iv). As the intracavernous internal carotid artery aneurysm demonstrated a less rupture potential can be conservatively treated if the size is less than $10 \mathrm{~mm}$.

(v). Immediate treatment will be adopted for aneurysms with irregular shape, unsmooth edge and/or daughter bubbles formation, which indicate high possibility of bleeding.

(vi). Untreated UIA should be re-examined every year, and immediate treatment will be adopted if the aneurysms enlarged or changed into high-risk shapes.

\section{CONCLUSION}

This initial clinical study substantiates the claim that one-stage endovascular embolization is a safe and effective method for treating multiple intracranial aneurysms. However, due to the small sample size used in this study, the long-term efficacy of one-stage embolization requires further evaluation.

\section{ACKNOWLEDGMENT}

This study was supported by the National High-tech R\&D Program of China (863 Program) (number 2015AA020301).
We would like to thank the native English speaking scientists of Elixigen Company (Huntington Beach, CA, USA) for editing our manuscript.

\section{- REFERENCES}

1. Cheong JJ, Ghinea N, van Gelder JM: Estimating the annual rate of de novo multiple aneurysms: Three statistical approaches. Neurosurg Focus 17(5):E8, 2004

2. Hino A, Fujimoto M, Iwamoto Y, Yamaki T, Katsumori T: False localization of rupture site in patients with multiple cerebral aneurysms and subarachnoid hemorrhage. Neurosurgery 46(4):825-830, 2000

3. Hoh BL, Chi YY, Lawson MF, Mocco J, Barker FG 2nd: Length of stay and total hospital charges of clipping versus coiling for ruptured and unruptured adult cerebral aneurysms in the Nationwide Inpatient Sample database 2002 to 2006. Stroke 41:337-342, 2010

4. Hong T, Wang Y: Unilateral approach to clip bilateral multiple intracranial aneurysms. Surg Neurol 72 (Suppl 1):S23-28, discussion S28, 2009

5. Inagawa T: Surgical treatment of multiple intracranial aneurysms. Acta Neurochir (Wien) 108:22-29, 1991

6. James Ling A, D'urso PS, Madan A: Simultaneous microsurgical and endovascular management of multiple cerebral aneurysms in acute subarachnoid haemorrhage. J Clin Neurosci 13(7):784-788, 2006

7. Juvela S: Risk factors for multiple intracranial aneurysms: Stroke 31: 392-397, 2000

8. Kaminogo M, Yonekura M, Shibata S: Incidence and outcome of multiple intracranial aneurysms in a defined population. Stroke 34: 16-21, 2003

9. Kassell NF, Torner JC, Jane JA, Haley EC Jr, Adams HP: The international cooperative study on the timing of aneurysm surgery. Part 2: Surgical results. J Neurosurg 73: 37-47, 1990

10. Matouk CC, Mandell DM, Günel M, Bulsara KR, Malhotra A, Hebert R, Johnson MH, Mikulis DJ, Minja FJ: Vessel wall magnetic resonance imaging identifies the site of rupture in patients with multiple intracranial aneurysms: Proof of principle. Neurosurgery 72(3): 492-496; discussion 496, 2013

11. Mizoi K, Suzuki J, Yoshimoto T: Surgical treatment of multiple aneurysms: Review of experience with 372 cases. Acta Neurochir (Wien) 96:8-14, 1989

12. Mount LA, Brisma R: Treatment of multiple intracranial aneurysms. J Neurosurg 35:728-730, 1971

13. Rinne J, Hernesniemi J, Niskanen M, Vapalahti M: Management outcome for multiple intracranial aneurysms. Neurosurgery 36: 31-37, discussion 37-38, 1995

14. Rinne J, Hernesniemi J, Puranen M, Saari T: Multiple intracranial aneurysms in a defined population: Prospective angiographic and clinical study. Neurosurg 35: 803-808, 1994

15. Roy D, Milot G, Raymond J: Endovascular treatment of unruptured aneurysms. Stroke 32(9): 1998-2004, 2001

16. Solander S, Ulhoa A, Uinuela F, Duckwiler GR, Gobin YP, Martin NA, Frazee JG, Guglielmi G: Endovascular treatment of multiple intracranial aneurysms by using Guglielmi detachable coils. J Neurosurg 90(5):857-864, 1999

17. Vajda J: Multiple intracranial aneurysms: A high risk condition. Acta Neurochir (Wien) 118:59-75, 1992 